\title{
Umpolung Addition of Aldehydes to Aryl Imines
}

\author{
Ning Chen ${ }^{+}$Xi-Jie Dai ${ }^{+}$, Haining Wang ${ }^{+}$, and Chao-Jun Li*
}

\begin{abstract}
One of the classical ways to synthesize amines involves the coupling of carbonyl compounds and imines, either through enolate chemistry or acyl-based carbanion equivalents. We herein report an alternative strategy that is based on the use of aldehydes as alkyl carbanion equivalents in a reductive coupling with aryl imines. A wide array of secondary amines can be synthesized in moderate to high yields. This reaction is mediated by hydrazine and catalyzed by ruthenium(II) complexes, and it tolerates various functional groups, such as esters, amides, and nitriles.
\end{abstract}

Amines are ubiquitous in natural products and bioactive molecules. Organic nitrogen compounds constitute over $90 \%$ of the 200 top-selling drugs, and feature in many name reactions. ${ }^{[1,2]}$ Over the past decades, tremendous efforts have been devoted to the synthesis of a wide array of amines. In particular, the direct nucleophilic addition of organometallic reagents (e.g., Grignard reagents) to imines represents a powerful synthetic method for such purposes. ${ }^{[3]}$ Despite the robustness of reactivity, most organometallic reagents are typically prepared from stoichiometric amounts of the corresponding metals and organic halides (Scheme 1a). Another obvious drawback associated with this high reactivity is the poor chemoselectivity, exemplified by low functional group compatibility towards, among others, esters, amides, and nitriles. Alternative strategies for amine synthesis include the Mannich reaction via enolate formation ${ }^{[4]}$ (Scheme $1 \mathrm{~b})$ as well as the umpolung addition of aldehydes to imines, for example in benzoin-type condensations (Scheme $1 \mathrm{c})^{[5,6]}$ and pinacol-type couplings (Scheme $\left.1 \mathrm{~d}\right) \cdot{ }^{[7,8]}$ All of these common alternatives feature oxygenated carbanions derived from aldehydes. ${ }^{[9]}$ Herein, we describe a novel umpolung addition of aldehydes to aryl imines for efficient amine synthesis, a mild process that is mediated by hydrazine in the presence of a ruthenium(II) complex (Scheme 1e).

Very recently, we developed a ruthenium-catalyzed, redox-based strategy for the direct deoxygenation of aliphatic primary alcohols in complex organic molecules with great efficiency and excellent selectivity. ${ }^{[10]}$ According to our

[*] Dr. N. Chen, ${ }^{[+]}$X.-J. Dai, ${ }^{[+]}$Dr. H. Wang, ${ }^{[+]}$Prof. Dr. C.-J. Li Department of Chemistry and FQRNT Centre for Green Chemistry and Catalysis, McGill University

801 Sherbrooke St. W., Montreal, Quebec H3A OB8 (Canada) E-mail: cj.li@mcgill.ca

Dr. N. Chen ${ }^{[+]}$

Department of Organic Chemistry, Faculty of Science

Beijing University of Chemical Technology

Beijing (China)

$\left.{ }^{+}\right]$These authors contributed equally to this work.

(1) Supporting information for this article can be found under: https://doi.org/10.1002/anie.201610578.
Nucleophilic addition of organometallic reagents to imines

$$
\mathrm{R}^{1} \mathrm{CH}_{2} \mathrm{X} \stackrel{[\mathrm{M}]}{\longrightarrow} \mathrm{R}^{1} \mathrm{CH}_{2} \mathrm{M} \underset{\mathrm{M}: \mathrm{Mg}, \mathrm{Zn}, \mathrm{Cu}, \mathrm{Li} \text {, etc. }}{\longrightarrow} \overbrace{\mathrm{R}^{1}}^{\mathrm{R}^{2} \mathrm{~N}_{-\mathrm{R}^{3}}} \stackrel{\mathrm{N}_{-}}{\mathrm{R}^{3}}
$$

Coupling of carbonyl compounds with imines: Mannich reaction

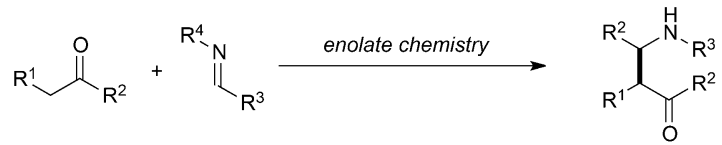

Umpolung coupling of aldehydes and imines

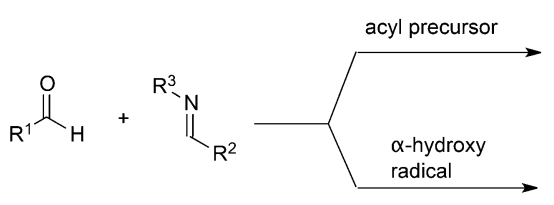<smiles>[R]NC([R])C([R])N[R]</smiles>

Aldehydes as alkyl carbanion equivalents for amine synthesis

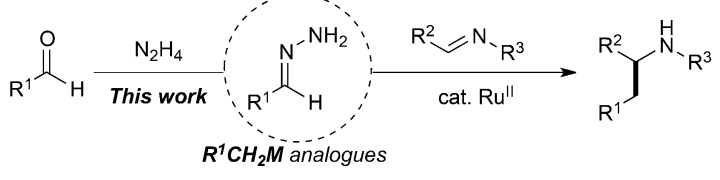

Scheme 1. Classical strategies for amine synthesis. a) Addition of organometallic reagents to imines. b-d) Addition of carbonyl-derived oxygenated carbanions to imines. e) Addition of aldehyde-derived alkyl carbanions to aryl imines.

proposed mechanism, the ruthenium(II)-coordinated hydrazone A (derived from a carbonyl compound) might react with another carbonyl compound to form a new carbon-carbon bond via a six-membered-ring transition state $\mathbf{B}$ (a Zimmerman-Traxler chair-like transition state; Scheme 2). This hypothesis was lately proven to be synthetically viable, providing rapid access to a broad range of secondary and tertiary alcohols. ${ }^{[1]}$ Encouraged by this proof-of-concept discovery, we questioned whether imines could be involved as alternative electrophilic partners in transition state $\mathbf{C}$. If so, the subsequent imine addition can be readily envisioned for amine synthesis. However, the much lower reactivity of imines combined with the potential complications arising from the structural similarity of imines and hydrazones rendered the development of such an addition reaction a great challenge.

In our model study, $N$-benzylidene aniline (2a) was chosen as the imine coupling partner while para-chlorobenzaldehyde (1a) was used as the carbanion precursor to be able to differentiate the nucleophilic partner from the electrophilic counterpart. Our initial attempts indicated that four major side products were generated over the course of the reaction, namely hydrazinolysis (aniline and azine), hydrolysis (benzaldehyde), and deoxygenated products (para-chlorotoluene; 


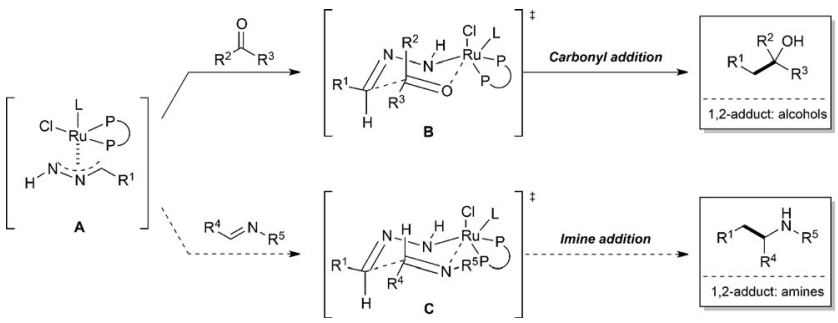

Scheme 2. Mechanistic hypothesis for the imine addition. Inspired by our proposed ruthenium complex $\mathbf{A}$ in the deoxygenation chemistry and the six-membered-ring chair-like transition state (TS) B in carbonyl addition, we surmised that a similar TS $\mathbf{C}$ could be invoked for the synthesis of amines from imines.

see the side products in Table 1). Subsequent catalyst screening suggested that ruthenium(II) catalysts with spectator ligands efficiently catalyzed the reaction, with $[\mathrm{Ru}(p$-cymene) $\left.\mathrm{Cl}_{2}\right]_{2}$ (the least expensive one) providing the highest yield (Table 1, entries 1-3). On the other hand, the evaluation of various phosphine ligands showed that the reaction was generally favored by electron-rich phosphines, such as 1,2bis(dimethylphosphino)ethane (dmpe) and tri(tert-butyl)phosphine (entries 5-7). It should be noted that the loading of the phosphine ligand has a strong influence on the reaction efficiency. Specifically, the yield was significantly lower when the ligand-to-metal ratio was increased to 2:1 (entry 5). This result implies that an excessive amount of phosphine ligand might saturate the ruthenium catalyst, leaving no empty coordination sites for the reactants. The base is also critical for this reaction as the desired product was not detected in the

Table 1: Optimization of the reductive coupling of aldehydes with imines.

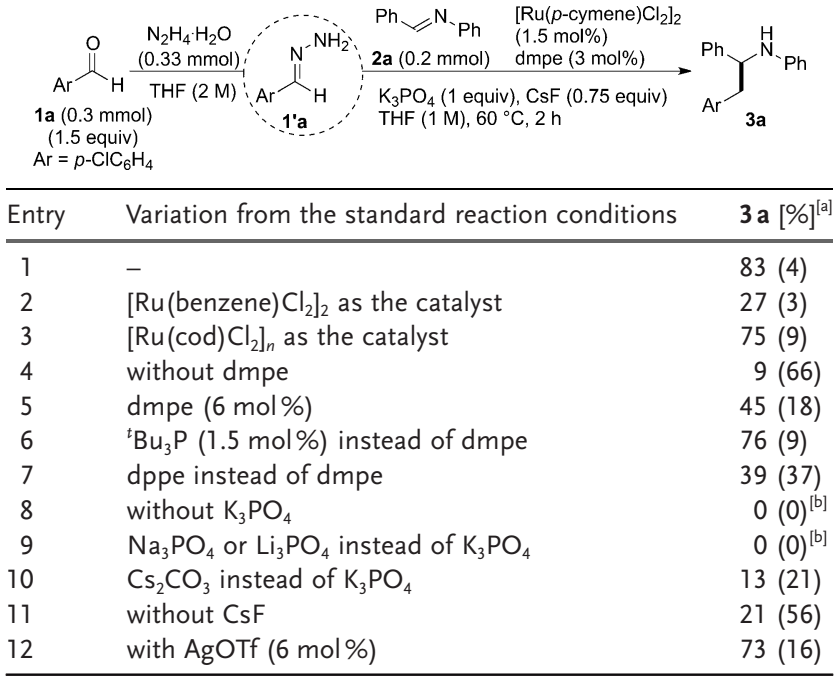

[a] All yields were determined by ${ }^{1} \mathrm{H}$ NMR analysis using trimethoxybenzene as an internal standard; the NMR yields of recovered unreacted $\mathbf{2} \mathbf{a}$ are given in parentheses. [b] Complete hydrazinolysis of $\mathbf{2} \mathbf{a}$ was observed. side products:

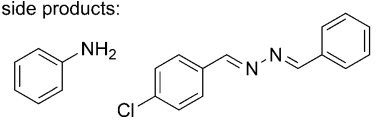<smiles>Cc1ccc(Cl)cc1</smiles>

absence of $\mathrm{K}_{3} \mathrm{PO}_{4}$ (entry 8). Intriguingly, whereas superior reactivity was observed with $\mathrm{K}_{3} \mathrm{PO}_{4}$, phosphates and carbonates bearing other alkali metal cations displayed no or little reactivity (entries 9 and 10). Furthermore, cesium fluoride turned out to be vital to increase the reaction efficiency (entry 11). Finally, the removal of a chloride from the ruthenium catalyst with silver triflate diminished the product yield (entry 12).

With optimized reaction conditions in hand, we then investigated the aldehyde scope (Scheme 3 ). To probe the generality of the current reaction, a variety of aryl-, heteroaryl-, and alkyl-substituted aldehydes were tested as nucleophilic partners in the coupling with $N$-benzylidene aniline (2a). In general, aromatic aldehydes showed good reactivity in this reaction. Specifically, different halide substituents on the arene only had a minor influence on the reactivity. In comparison with benzaldehyde, similar yields were obtained with chlorine or fluorine substituents at the para position (Scheme 3, $\mathbf{3 a}$ and $\mathbf{3 i}$ vs. 3b). Compared to meta- and parasubstituted chlorobenzaldehyde, the ortho-substituted one was the least reactive (3d vs. $3 \mathbf{a}$ and $\mathbf{3 c}$ ). These results indicate that the reaction is strongly influenced by steric hindrance. Similar observations for steric bias were made with para-, meta-, and ortho-tolualdehyde (3e-3 g). Further studies with para-substituted aldehydes showed that electronic effects play an insignificant role in this reaction as substrates

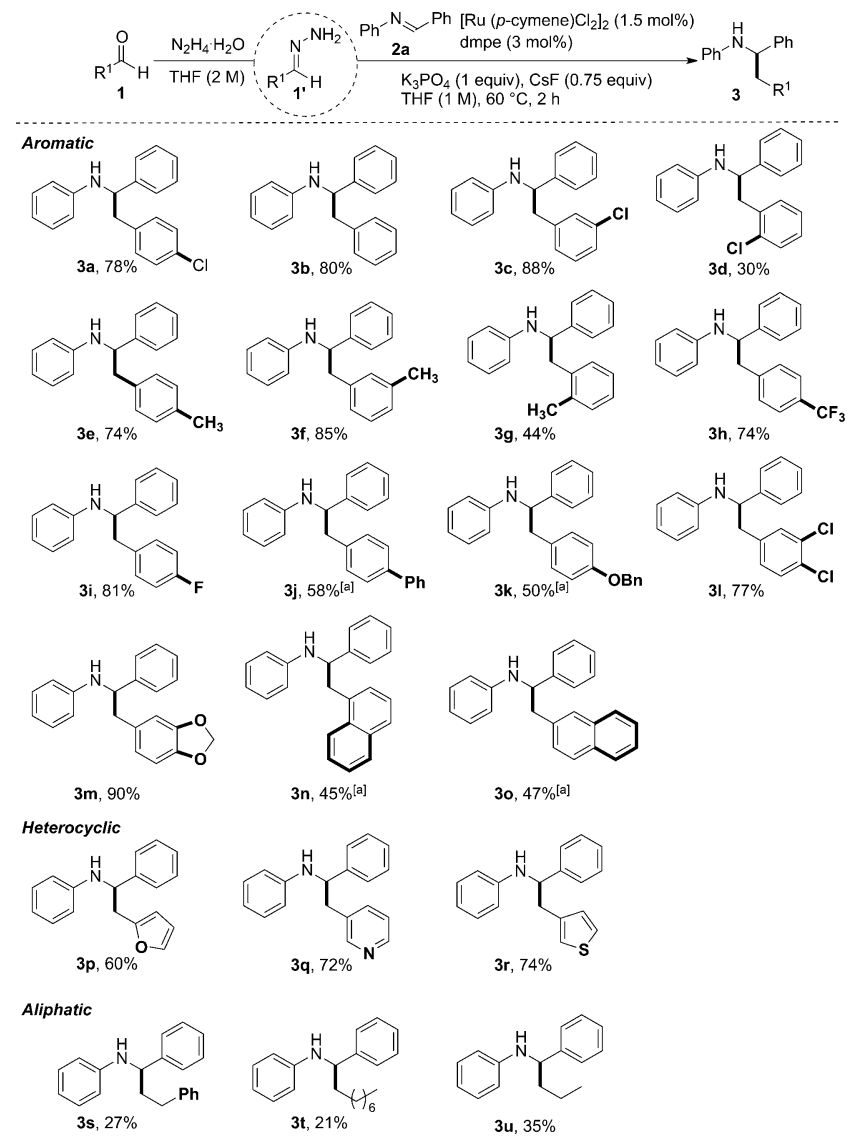

Scheme 3. Aldehyde scope. Reaction conditions: 2 a $(0.2 \mathrm{mmol})$, 1 ( $0.3 \mathrm{mmol}, 1.5$ equiv). Yields of isolated products are given. [a] The hydrazones were separated by simple filtration prior to use. 
with either strongly electron-withdrawing $\left(\mathrm{CF}_{3}, \mathbf{3 h}\right.$; 3,4dichloro, 3I) or strongly electron-donating groups (dioxo, $\mathbf{3 m}$ ) provided comparable yields. Hydrazones prepared from benzaldehydes with phenyl, benzyloxy, or naphthyl substituents were poorly soluble in THF (1'j, $\mathbf{1}^{\prime} \mathbf{k}, \mathbf{1}^{\prime} \mathbf{n}$, and $\left.\mathbf{1}^{\prime} \mathbf{o}\right)$. In these cases, the corresponding hydrazones were separated by a simple filtration because of their facile precipitation. As a result, the direct use of these solid hydrazones $\left(\mathbf{1}^{\prime}\right)$ afforded moderate yields of the corresponding amines $(\mathbf{3} \mathbf{j}, \mathbf{3} \mathbf{k}, \mathbf{3} \mathbf{n}$, and 3o). In cases where low yields were obtained, most of the imine substrate $\mathbf{2 a}$ was recovered (3d, 3g, and $\mathbf{3 n})$. The couplings of heteroaromatic aldehydes, such as 2-furan-, 3-pyridine-, and 3-thiophenecarboxaldehyde, proceeded well to give the corresponding products in good yields $(\mathbf{3} \mathbf{p}-\mathbf{3 r} \mathbf{r})$. Finally, aliphatic aldehydes were also effective in this reaction but the desired products were formed in lower yields owing to competing elimination reactions $(\mathbf{3} \mathbf{s}-\mathbf{3} \mathbf{u})$.

Having established the scope of the reaction with respect to the nucleophilic partner, we moved on to investigate the suitability of various electrophilic imines (Scheme 4). A wide range of substituted aromatic imines were coupled with parachlorobenzylidene hydrazine (1'a) or benzylidene hydrazine $\left(\mathbf{1}^{\prime} \mathbf{b}\right)$ in moderate to good yields. We identified a mild electronic influence on the reactivity upon varying the aromatic substituent on the imine carbon atom. For example, the yield dropped from $82 \%$ to $56 \%$ when an electronwithdrawing fluoro substituent was replaced with an electrondonating 1,3-dioxole substituent (4c and $\mathbf{4} \mathbf{f}$ ). This result may be explained in terms of the decreased electrophilicity of the corresponding imine. By contrast, a dramatic electronic influence was observed for variations of the aromatic substituent on the imine nitrogen atom. Compared with $\mathbf{4 d}$, 4i, which features a para-methyl instead of a hydrogen substituent, was formed in significantly lower yield. With $N$-piperonylidene (para-tolyl)amine $(\mathbf{2} \mathbf{j})$, a very electron-rich imine, the yield even dropped to only $10 \%$. To our delight, upon modifying the reaction conditions by adding hydrazone 1'a in five portions (with a 30 minute interval), the yield was improved to $44 \%(\mathbf{4 j})$. The yields also improved when a chlorine substituent, as a weakly electron-withdrawing group, was attached to the aromatic ring on the imine nitrogen atom $(\mathbf{4 k}-\mathbf{4 m})$. We also found that the coupling of an imine with a naphthalene-1-yl group at the nitrogen atom $(4 \mathbf{n}, 60 \%)$ is slightly more efficient than that of the corresponding imine with the naphthalene-1-yl group at the imine carbon atom $(\mathbf{4 h}, 47 \%)$. These results confirmed that the steric influence of substituents on the imine carbon atom is stronger than that resulting from substituents on the nitrogen atom. Notably, sensitive functional groups that are incompatible with robust organometallic reagents, such as esters, amides, and nitriles, remained untouched in this process. Similarly, the poor reactivity of $\mathbf{4 p}$ is likely due to the electron-donating nature of the acetamido group.

Dibenzoxazepines, seven-membered aza heterocycles featuring cis imines, are commonly found in a large number of bioactive molecules. Many of them display interesting physiological activities and can be used as antidepressants, antipsychotics, and HIV-1 reverse transcriptase inhibitors. ${ }^{[12,13]}$ Three dibenzo[b,f][1,4] oxazepine derivatives $(\mathbf{2}$ s-

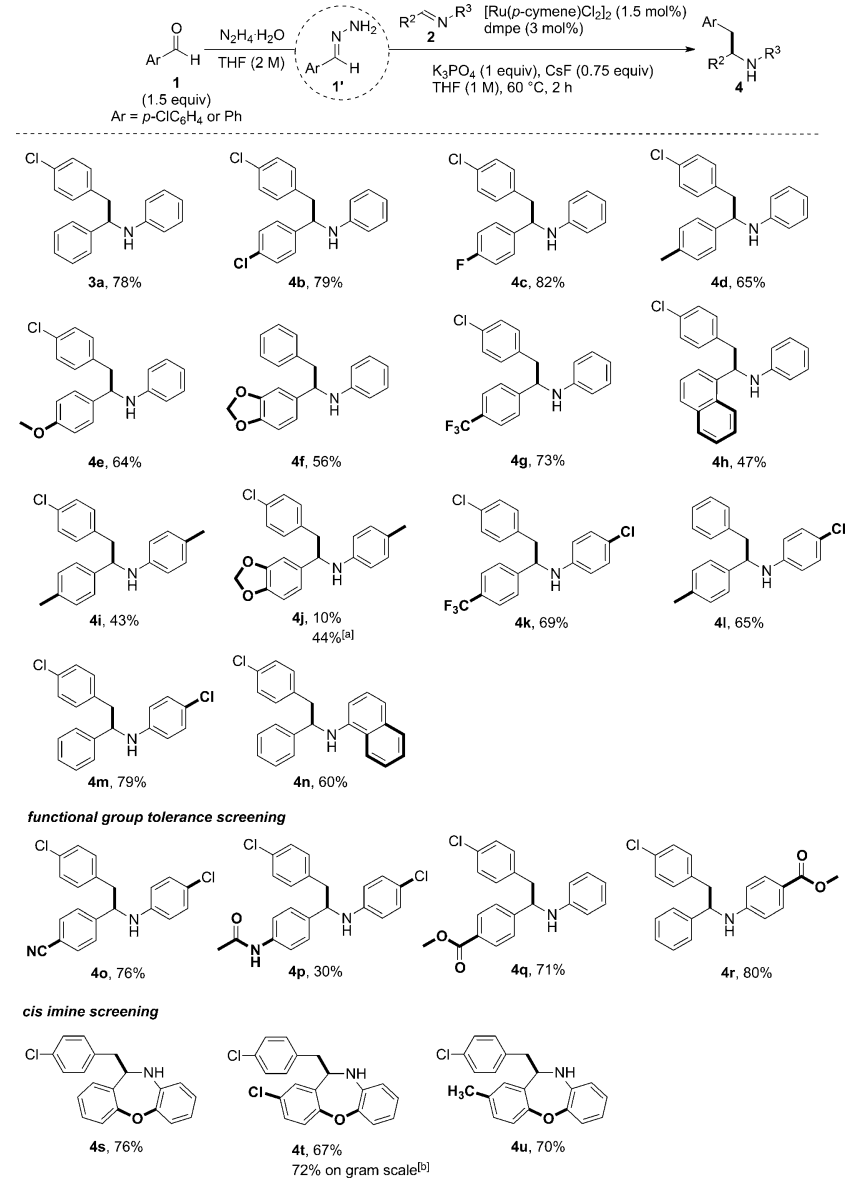

Scheme 4. Imine scope. Yields of isolated products are given. Reactions conditions: 2 a $(0.2 \mathrm{mmol}), 1$ a or $1 \mathbf{b}(0.3 \mathrm{mmol}, 1.5$ equiv). [a] The hydrazone was added in five portions with 30 min intervals ( $2.5 \mathrm{~h}$ in total). [b] The gram-scale reaction was performed using $2 \mathrm{t}$ $(4 \mathrm{mmol})$ and $1 \mathrm{a}(6 \mathrm{mmol})$ in the presence of $\left[\mathrm{Ru}(p \text {-cymene }) \mathrm{Cl}_{2}\right]_{2}$ (0.75 mol\%) and dmpe (1.5 mol\%).

$2 \mathbf{u})$ were examined in the current process, and all afforded the corresponding products in good yields (Scheme 4, 4s-4u). Unfortunately, aliphatic imines such as $N$-propylidene aniline, $N$-benzylidene methanamine, and $N$-benzylidene benzylamine, as well as ketimines such as $N, 1$-diphenylethan-1-imine were comparatively inert under the standard conditions.

Finally, this method was assessed on a larger scale. Gratifyingly, $1.03 \mathrm{~g}$ of $\mathbf{4 t}$ were obtained from $0.92 \mathrm{~g}$ of imine 2t $(4 \mathrm{mmol})$ at $60^{\circ} \mathrm{C}$, with only $0.75 \mathrm{~mol} \%$ of $\mathrm{Ru}(p$-cymene) $\left.\mathrm{Cl}_{2}\right]_{2}$ and $1.5 \mathrm{~mol} \%$ of dmpe, which corresponds to a yield of $72 \%$ (Scheme $4, \mathbf{4 t}$ ). To facilitate this scale-up manipulation, a three-neck round-bottom flask with a condenser equipped with a nitrogen balloon was used as an alternative reaction vessel.

In summary, we have developed a method for the umpolung addition of aldehydes to aryl imines that is mediated by hydrazine in the presence of a ruthenium(II) precatalyst and dmpe. This process features aldehyde-derived alkyl carbanion equivalents, complementary to the oxygenated carbanions used in traditional coupling reactions between carbonyl compounds and aryl imines. Compared to classical organometallic coupling reactions, this approach not 
only bypasses the use of stoichiometric quantities of metals and organic halides, but also tolerates various sensitive functional groups, such as amides, esters, and nitriles. The scope and mechanism of this method as well as applications in total synthesis are currently under investigation.

\section{Acknowledgements}

This work was financially supported by the Canada Research Chair (Tier 1) foundation, FQRNT (CCVC), NSERC, CFI, and McGill University. We also thank the CSC (China Scholarship Council) for a postdoctoral fellowship (N.C.). We sincerely thank the group of Prof. Jiaxi $\mathrm{Xu}$ at the Beijing University of Chemical Technology for providing the cis imines $(\mathbf{2} \mathbf{s}-\mathbf{2 u})$.

\section{Conflict of interest}

The authors declare no conflict of interest.

Keywords: aldehydes · hydrazine - imines · reductive addition . ruthenium catalysis

How to cite: Angew. Chem. Int. Ed. 2017, 56, 6260-6263 Angew. Chem. 2017, 129, 6356-6359

[1] For the top 200 pharmaceutical drugs from 2007 to 2013, see a) E. A. Ilardi, E. Vitaku, J. T. Njardarson, J. Chem. Educ. 2013 90, 1403; b) E. Vitaku, D. T. Smith, J. T. Njardarson, J. Med. Chem. 2014, 57, 10257.

[2] J. J. Li, Name Reactions: A Collection of Detailed Mechanisms and Synthetic Applications, Springer, New York, 2006.

[3] For selected reviews, see: a) K.-J. Yamada, K. Tomioka, Chem. Rev. 2008, 108, 2874; b) S. Kobayashi, Y. Mori, J. S. Fossey, M. M. Salter, Chem. Rev. 2011, 111, 2626; c) V. N. G. Lindsay, A. B. Charette in Comprehensive Organic Synthesis II, 2nd ed. Elsevier, Amsterdam, 2014, p. 365; d) J. H. Kim, Y. O. Ko, J. Bouffard, S.-G. Lee, Chem. Soc. Rev. 2015, 44, 2489; e) N Kumagai, M. Shibasaki, Bull. Chem. Soc. Jpn. 2015, 88, 503; f) F. Stefania, Tetrahedron 2016, 72, 4449; for selected recent examples, see: g) A. Das, R. Alam, L. Eriksson, K. J. Szabó, Org. Lett. 2014, 16, 3808; h) S. Hirner, A. Kolb, J. Westmeier, S. Gebhardt, S. Middel, K. Harms, P. von Zezschwitz, Org. Lett. 2014, 16, 3162; i) A. Desmarchelier, P. Ortiz, S. R. Harutyunyan, Chem. Commun. 2015, 51, 703; j) M. Hatano, K. Yamashita, M. Mizuno, O. Ito, K. Ishihara, Angew. Chem. Int. Ed. 2015, 54, 2707; Angew. Chem. 2015, 127, 2745; k) T. Beisel, G. Manolikakes, Org. Lett. 2015, 17, 3162; 1) T. Jiang, Z. Wang, M.-H. Xu, Org. Lett. 2015, 17, 528; m) Y. Yang, I. B. Perry, S. L. Buchwald, J. Am. Chem. Soc. 2016, 138, 9787; n) T. Soeta, T. Ishizaka, Y. Ukaji, J. Org. Chem. 2016, 81, 2817.

[4] a) C. Mannich, W. Krösche, Arch. Pharm. 1912, 250, 647; b) J. M. M. Verkade, L. J. C. von Hemert, P. J. L. M. Quaedflieg, F. P. J. T. Rutjes, Chem. Soc. Rev. 2008, 37, 29; c) R. G. Arrayás, J. C. Carretero, Chem. Soc. Rev. 2009, 38, 1940.
[5] The umpolung of aldehydes to give acyl anion equivalents is normally applied in benzoin and Stetter reactions; see: a) X. Bugaut, F. Glorius, Chem. Soc. Rev. 2012, 41, 3511; b) D. M. Flanigan, F. Romanov-Michailidis, N. A. White, T. Rovis, Chem. Rev. 2015, 115, 9307; c) an earlier umpolung of aldehydes to carbanions via a hindered hydazone lithium salt: J. E. Baldwin, R. Aldlington, J. C. Bottaro, J. N. Kolhe, I. M. Newington, M. W. D. Perry, Tetrahedron, 1986, 42, 4235.

[6] For benzoin-type condensation with imines, see: a) G.-Q. Li, L.X. Dai, S.-L. You, Chem. Commun. 2007, 852; b) T. Hashimoto, M. Hirose, K. Maruoka, J. Am. Chem. Soc. 2008, 130, 7556; c) D. A. DiRocco, T. Rovis, Angew. Chem. Int. Ed. 2012, 51, 5904; Angew. Chem. 2012, 124, 6006; d) L.-H. Sun, Z.-Q. Liang, W.-Q. Jia, S. Ye, Angew. Chem. Int. Ed. 2013, 52, 5803; Angew. Chem. 2013, 125, 5915.

[7] For reviews on the umpolung of aldehydes to give $\alpha$-hydroxy radicals, which is normally applied in pinacol couplings, see: a) $\mathrm{T}$. Hirao, Top. Curr. Chem. 2007, 279, 53; b) M. Szostak, N. J. Fazakerley, D. Parmar, D. J. Procter, Chem. Rev. 2014, 114, 5959.

[8] For pinacol-type couplings with imines, see: a) V. Faugeroux, Y. Genisson, Curr. Org. Chem. 2008, 12, 751; b) E. J. Roskamp, S. F. Pedersen, J. Am. Chem. Soc. 1987, 109, 6551; c) F. Machrouhi, J.L. Namy, Tetrahedron Lett. 1999, 40, 1315; d) T. Tsukinoki, S. Nagashima, Y. Mitoma, M. Tashiro, Green Chem. 2000, 2, 117; e) M. Shimizu, A. Iwata, H. Makino, Synlett 2002, 1538; f) H. Maekawa, Y. Yamamoto, H. Shimada, K. Yonemura, I. Nishiguchi, Tetrahedron Lett. 2004, 45, 3869; g) L. Keinicke, P. Fristrup, P. Norrby, R. Madsen, J. Am. Chem. Soc. 2005, 127, 15756; h) J. L. Chiara, Á. García, Synlett 2005, 2607; i) N. Kise, A. Yamane, S. Nakao, A. Takebe, T. Sakurai, Tetrahedron: Asymmetry 2011, 22, 1906; j) G. Fan, Y. Liu, Tetrahedron Lett. 2012, 53, 5084; k) R. Umeda, M. Ninomiya, T. Nishino, M. Kishida, S. Toiya, T. Saito, Y. Nishiyama, N. Sonoda, Tetrahedron 2015, 71, 1287.

[9] A. T. Biju, N. Kuhl, F. Glorius, Acc. Chem. Res. 2011, 44, 1182.

[10] a) X.-J. Dai, C.-J. Li, J. Am. Chem. Soc. 2016, 138, 5433; for a book on ruthenium-catalyzed reactions, see: b) C. Bruneau, P. H. Dixneuf, Ruthenium Catalysts and Fine Chemistry, Springer Verlag, Heidelberg, 2004.

[11] H. Wang, X.-J. Dai, C.-J. Li, Nat. Chem. 2016, DOI: 10.1038/ NCHEM.2677.

[12] Amoxapine and loxapine, typical dibenzoxazepine derivatives, are known antidepressants and antipsychotics; see: a) R. C. Heel, R. N. Brogden, T. M. Speight, G. S. Avery, Drugs 1978, 15, 198; b) S. G. Jue, G. W. Dawson, R. N. Brogden, Drugs 1982, 24 , 1; for other bioactivity studies, see: c) J. M. Klunder, K. D. Hargrave, M. West, E. Cullen, K. Pal, M. L. Behnke, S. R. Kapadia, D. W. McNeil, J. C. Wu, G. C. Chow, J. Med. Chem. 1992, 35, 1887; d) H. J. M. Gijsen, D. Berthelot, M. Zaja, B. Brône, I. Geuens, M. Mercken, J. Med. Chem. 2010, 53, 7011.

[13] For the application of dibenzoxazepines in organic synthesis, see: a) L. Jiao, Y. Liang, J.-X. Xu, J. Am. Chem. Soc. 2006, 128, 6060 ; b) K. Gao, C.-B. Yu, W. Li, Y.-G. Zhou, X. Zhang, Chem. Commun. 2011, 47, 7845; c) C. Deraeve, Z. Guo, R. S. Bon, W. Blankenfeldt, R. DiLucrezia, A. Wolf, S. Menninger, E. A. Stigter, S. Wetzel, A. Choidas, K. Alexandrov, H. Waldmann, R. S. Goody, Y.-W. Wu, J. Am. Chem. Soc. 2012, 134, 7384.

Manuscript received: October 28, 2016

Final Article published: December 27, 2016 